Pierre-Alain Duc, Jonathan Braine and Elias Brinks, eds.

\title{
Discovery of Intergalactic H II Regions
}

\author{
E. V. Ryan-Weber \\ University of Melbourne, Australia
}

M. E. Putman

CASA, University of Colorado, USA

K. C. Freeman

RSSA, Australian National University, Australia

G. R. Meurer

The Johns Hopkins University, USA

R. L. Webster

University of Melbourne, Australia

\begin{abstract}
We have discovered a number of very small isolated H II regions $20-30 \mathrm{kpc}$ from their nearest galaxy. The Hil regions appear as tiny emission line dots (ELdots) in narrow band images obtained by the NOAO Survey for Ionization in Neutral Gas Galaxies (SINGG). We have spectroscopic confirmation of 5 isolated $\mathrm{H}$ in regions in 3 systems. The $\mathrm{H} \alpha$ luminosities of the $\mathrm{H}$ II regions are equivalent to the ionizing flux of only 1 large or a few small OB stars each. These stars appear to have formed in situ and represent atypical star formation in the low density environment of galaxy outskirts. In situ star formation in the intergalactic medium offers an alternative to galactic wind models to explain metal enrichment. In interacting systems (2 out of 3 ), isolated $\mathrm{H}$ II regions could be a starting point for tidal dwarf galaxies.
\end{abstract}

\section{Introduction}

Isolated $\mathrm{H}$ II regions in the extreme outskirts of galaxy halos (Gerhard et al., 2002) and in gaseous tidal debris (Ryan-Weber et al., 2003c; Oosterloo et al., 2003) have recently been discovered. Isolated $\mathrm{H}$ II regions indicate the formation of OB stars in atypical environments. Their existence poses questions about the conditions required to form stars. Star formation usually occurs in the inner parts of galaxies and is aided by a high density of gas that is unstable against gravitational collapse and shielded from the extragalactic ionizing background. 
Here we discuss five small isolated $\mathrm{H}$ II regions. The $\mathrm{H}$ II regions were discovered in the NOAO Survey for Ionization in Neutral Gas Galaxies (SINGG). SINGG is an $\mathrm{H} \alpha$ survey of an $\mathrm{H}$ I-selected sample of nearby galaxies. The survey is composed of nearly 500 galaxies from the H I Parkes All-Sky Survey (HIPASS, Meyer et al., 2003), of these about 300 have been observed in $\mathrm{H} \alpha$. The H II regions appear as tiny Emission Line dots (ELdots) at projected distances up to $30 \mathrm{kpc}$ from the apparent host galaxy and at least beyond two $R_{25}$ (R-band isophotal radii with $\mu_{R}=25 \mathrm{mag} \operatorname{arcsec}^{-2}$ ). This is typically much further from the apparent host than outer disk H II regions in spirals (Ferguson, 1998b). Isolated H II regions are described as 'intergalactic' as they lie well beyond the main optical radius of the nearest galaxy, but may or may not be kinematically bound to it.

\section{Observations}

Continuum R-band and narrow band $\mathrm{H} \alpha$ images of local gas-rich galaxies were taken with the CTIO $1.5 \mathrm{~m}$ telescope as part of SINGG. The candidate isolated $\mathrm{H}$ II regions were identified as unresolved high equivalent width (EW) sources outside the optical disk of each galaxy. The isolated $\mathrm{H}$ II regions have $\mathrm{H} \alpha$ fluxes in the range $9.7 \times 10^{-16}$ to $2.1 \times 10^{-15} \mathrm{erg} \mathrm{s}^{-1} \mathrm{~cm}^{-2}$. Assuming the distance to the isolated $\mathrm{H}$ II regions is the same as that of the host galaxy in each system, the $\mathrm{H} \alpha$ luminosities are in the range $6.9 \times 10^{36}$ to $3.5 \times 10^{38} \mathrm{erg} \mathrm{s}^{-1}$. In most cases the isolated $\mathrm{H}$ II regions are barely detected in continuum emission in the SINGG R-band images with a typical $5 \sigma$ detection limit of $\sim 10^{-18} \mathrm{erg} \mathrm{s}^{-1} \mathrm{~cm}^{-2} \AA^{-1}$.

Spectra of isolated H II region candidates were obtained with the double beam spectrograph (DBS) on the RSAA 2.3m telescope in September 2002. The 5 detected isolated $\mathrm{H}$ II regions were confirmed as $\mathrm{H} \alpha(6563 \AA)$ emission line systems, with recessional velocities close to that of their respective host galaxies. Some isolated H II regions were also detected in [O III] (5007 ̊). The presence of both $\mathrm{H} \alpha$ and [O III] lines places the isolated $\mathrm{H}$ II regions at comparable recessional velocities to the galaxy (or galaxies) in each field, and rules out the possibility that they are background emission line systems. In addition to the $\mathrm{H} \alpha$ images and spectra, Australia Telescope Compact Array (ATCA) H I maps are available for two systems, NGC 1533 and ESO 149-G003. The ATCA data reduction is detailed in $\mathrm{Ryan}-$ Weber et al. (2003b).

\section{Three Systems with Confirmed Isolated H II Regions}

\subsection{NGC 1533}

Figure 1 shows a DSS image of NGC 1533 overlaid with ATCA H I contours. The $\mathrm{H}$ I distribution around NGC 1533 consists of two major arcs, the NW cloud and the SE cloud. No obvious optical counterpart to this $\mathrm{H}$ I feature is seen in the DSS nor R band SINGG image. The total H I mass of the system is $7 \times 10^{9} \mathrm{M}_{\odot}$. The SE cloud contains around one third of this total H I mass. The projected distance between the $\mathrm{HI}$ arcs and the optical centre of NGC 1533 ranges from $2^{\prime}$ to $11.7^{\prime}$ (12 and $70 \mathrm{kpc}$ ). The velocities of the three confirmed isolated $\mathrm{H}$ II regions compare well with the velocity of NGC 1533 and lie within the range of 


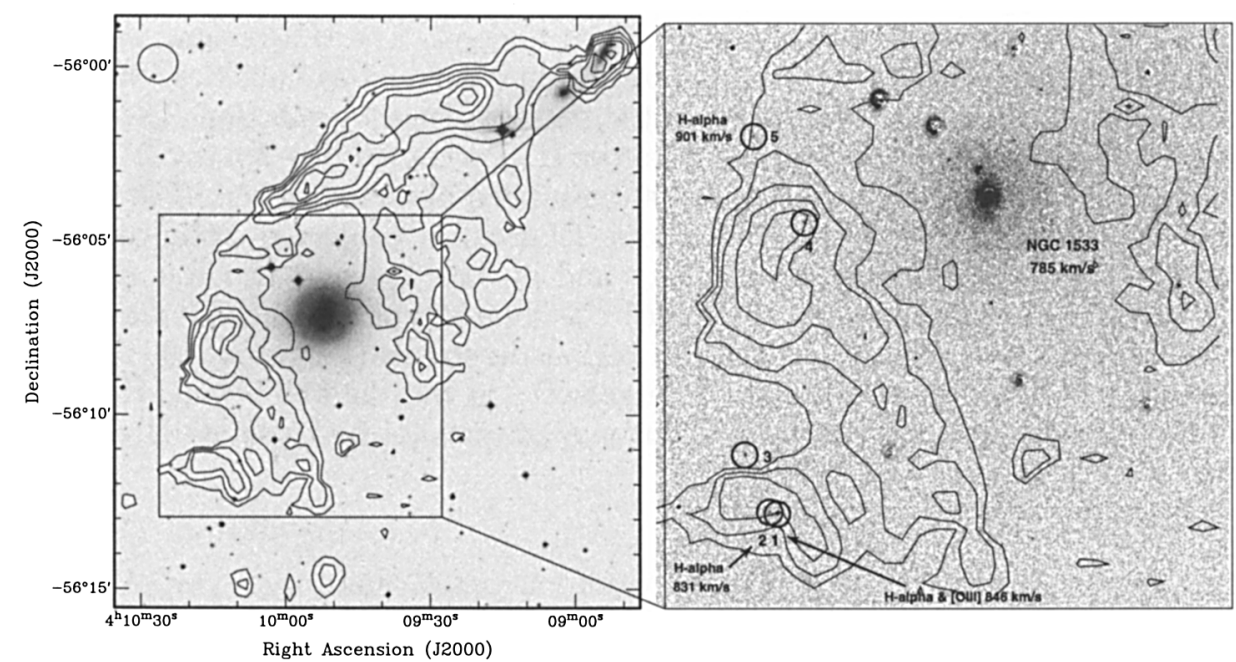

Figure 1. NGC 1533: DSS image with ATCA H I contours overlaid at 1.6, 2.0,2.4, 2.8,3.2, 3.6 and $4 \times 10^{20} \mathrm{~cm}^{-2}$. The beam is given in the top left corner. The insert shows the continuum subtracted $\mathrm{H} \alpha$ image with the isolated $\mathrm{H}$ II regions labelled and $\mathrm{H} \alpha$ velocities given.

$\mathrm{H}$ I velocities in the SE cloud $\left(883 \mathrm{~km} \mathrm{~s}^{-1}\right.$ with a width at $50 \%$ peak, $w_{50}=71$ $\mathrm{km} \mathrm{s}^{-1}$ ). The peculiar distribution of $\mathrm{HI}$ is thought to arise from the destruction of a galaxy to form a tidal remnant around NGC 1533. N-body/SPH numerical simulations showing the orbital evolution of a LSB galaxy in NGC 1533's gravitational potential support this hypothesis Ryan-Weber (2003a).

Are these isolated H II regions progenitors to a tidal dwarf galaxy? Since the gas and isolated H II regions are bound to the galaxy, it is likely that the stars formed will also remain bound in the tidal debris. There is certainly a reservoir of gas from which more stars could form, so it is possible in this case that a tidal dwarf galaxy could emerge. Interestingly, the isolated $\mathrm{H}$ II regions do not appear to be correlated with the densest regions of $\mathrm{HI}$ and are located in the SE cloud only. At this resolution $(\sim 6 \mathrm{kpc})$ the densest region of $\mathrm{HI}$ is the center the NW cloud. The stellar concentration of a tidal dwarf galaxy is usually located in the densest regions of $\mathrm{HI}$, mapped in $21-\mathrm{cm}$ at similar resolutions ( $4 \mathrm{kpc}$, e.g. Duc et al. 2000).

\subsection{HCG 16}

The isolated H II region in the compact group HCG 16, shown in Figure 2, is near the two galaxies NGC 835 and NGC 833. The velocity of the isolated $\mathrm{H}$ II region $\left(3634 \mathrm{~km} \mathrm{~s}^{-1}\right)$ sits on the lower edge of the $\mathrm{H} \mathrm{I}$ emission measured by HIPASS (velocity $=3917 \mathrm{~km} \mathrm{~s}^{-1}, w_{50}=288 \mathrm{~km} \mathrm{~s}^{-1}$ ) and below the optical velocities of NGC 835 and NGC 833 (4073 and $3864 \mathrm{~km} \mathrm{~s}^{-1}$ respectively, from NASA/IPAC Extragalactic Database, NED). Verdes-Montenegro et al. (2001) have published a VLA map of HCG 16, showing H I in NGC 835 and 833 with a 


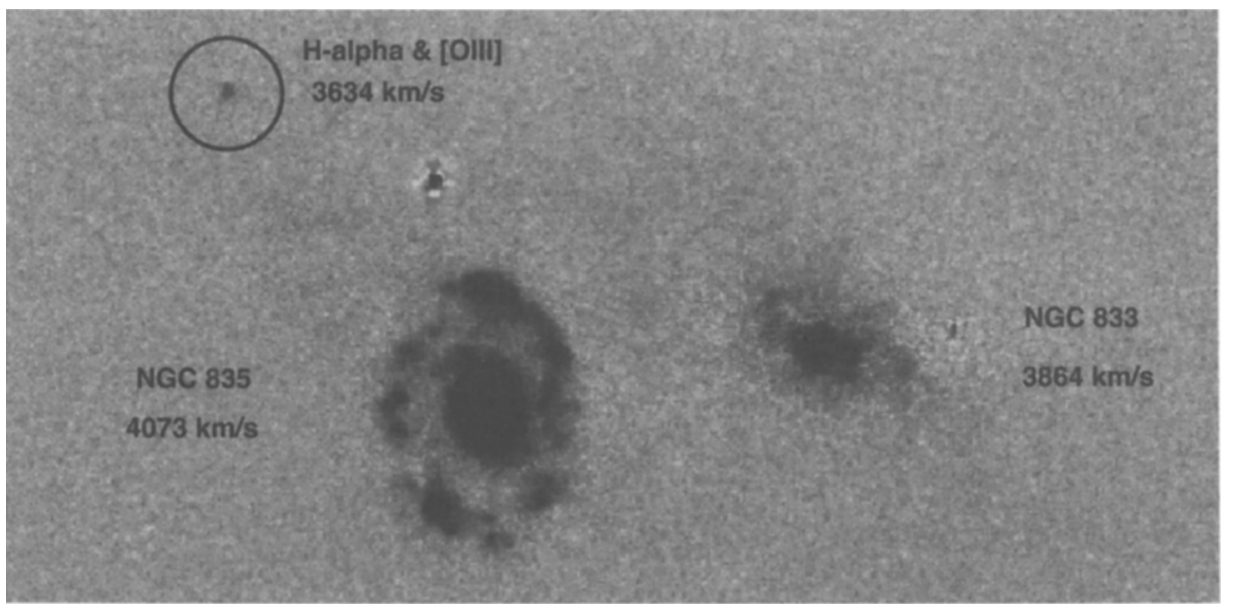

Figure 2. HCG 16: Continuum subtracted $\mathrm{H} \alpha$ image with the isolated $\mathrm{H}_{\mathrm{II}}$ region and two members of the galaxy group labelled. The other object in the field is residuals of a foreground star.

large tidal feature to the $\mathrm{NE}$ (overlapping the isolated $\mathrm{H}$ II region position) that joins other group members several arcminutes away to the east.

\subsection{ESO 149-G003}

The velocity of the isolated H II region near the irregular galaxy ESO 149-G003 (see Figure 3) is quite offset from its apparent host galaxy $\left(314 \mathrm{~km} \mathrm{~s}^{-1}\right.$, assuming the single emission line is indeed $\mathrm{H} \alpha)$. The narrow $\left(w_{50}=39 \mathrm{~km} \mathrm{~s}^{-1}\right) \mathrm{H} \mathrm{I}_{\mathrm{I}}$ spectrum of the galaxy shows no anomalous velocity gas. Follow-up ATCA observations show no $\mathrm{HI}$ emission at the velocity and position of the isolated $\mathrm{H}$ II region to a $3 \sigma$ limit, assuming a distance of $6.5(12) \mathrm{Mpc}$ of $\mathrm{M}_{\mathrm{HI}}=8 \times 10^{5}\left(3 \times 10^{6}\right)$ $\mathrm{M}_{\odot}$. The spatial and kinematic evidence suggests that the isolated $\mathrm{H}$ II region is perhaps a chance object in the field, rather than an associate of ESO 149G003. If the emission line is $\mathrm{H} \alpha$, this object holds interesting implications for the census of intergalactic matter. It could be sub-galactic halo forming its first generation of stars. However, since only one emission line $(\mathrm{H} \alpha)$ is detected in this case, the possibility that this candidate isolated $\mathrm{H}$ II region is a background emission line source cannot be ruled out, for example $\mathrm{H} \beta$ at $\mathrm{z} \sim 0.3$ or [O III] at $\mathrm{z} \sim 0.2$.

\section{Underlying Stellar Population}

The $\mathrm{H} \alpha$ luminosities indicate that each isolated $\mathrm{H}$ II region is illuminated by the equivalent of $0.2-8$ O5V stars each (Vacca et al. 1996). The least luminous isolated $\mathrm{H}$ II region could be ionized by a single $\mathrm{O} 8$ star. The isolated $\mathrm{H}$ II regions could be ionized by a single massive star, or by the massive star or stars in a cluster. The very low continuum emission does rule out a significant underlying stellar population and suggests that isolated $\mathrm{H}$ II regions are due to newly formed 


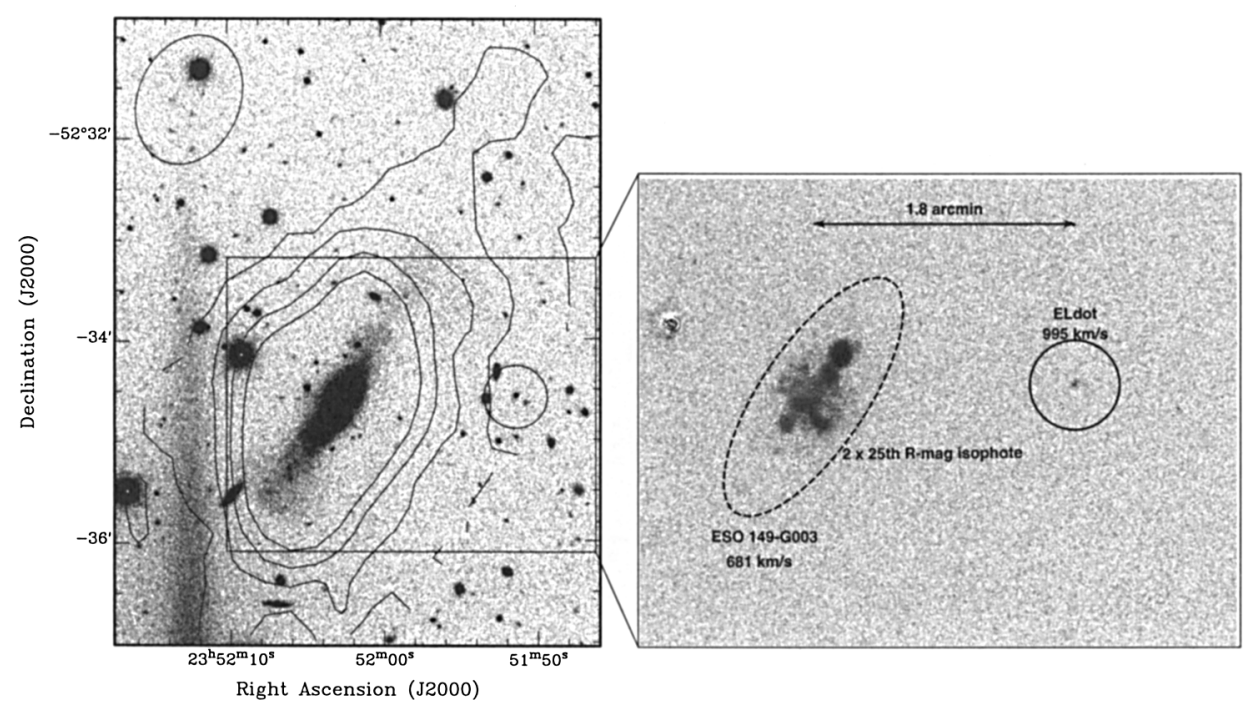

Figure 3. ESO 149-G003: R-band image with ATCA H I contours overlaid at $0.5,1.0,1.5$ and $2.0 \times 10^{20} \mathrm{~cm}^{-2}$. The beam is given in the top left corner. The insert shows the continuum subtracted $\mathrm{H} \alpha$ image with the isolated $\mathrm{H}$ II region and galaxy labelled.

clusters where no stars existed previously. The low continuum emission also separates isolated $\mathrm{H}$ II regions from $\mathrm{H}$ II galaxies and tidal dwarf galaxies. Three of the 5 isolated $\mathrm{H}$ II regions have $\mathrm{EW}(\mathrm{H} \alpha)>1000 \AA$. By comparison, $\mathrm{H}$ II regions in the outer arms of spiral galaxies (beyond the B-band 25th-magnitude isophote) measured by Ferguson (1998a) have an average $\mathrm{EW}(\mathrm{H} \alpha)=364 \AA$. Furthermore, most of the group of star forming dwarf galaxies in $\mathrm{A} 1367$ have $\mathrm{EW}(\mathrm{H} \alpha)<100 \AA$ (Sakai et al., 2002).

\section{IGM enrichment}

As they evolve, OB stars increase the metal abundance in their local environment. Absorption line studies show that the intergalactic medium (IGM) and galaxy halos, including our own, are enriched (e.g., Tripp et al., 2002; Collins et al., 2003). Isolated H II regions provide a potential source for this enrichment. In situ star formation in the IGM offers an alternative to galactic wind models to explain metal enrichment hundreds of kilo-parsecs from the nearest galaxy.

Although the $\mathrm{H} \alpha$ luminosities are small, an estimate of the Star Formation Rate (SFR) can be obtained by a standard relation (Kennicutt, 1998). Summing the $\mathrm{H} \alpha$ luminosities from the 5 isolated $\mathrm{H}$ II regions in the NGC 1533 system, the SFR $=3 \times 10^{-3} \mathrm{M}_{\odot} \mathrm{yr}^{-1}$. The total yield of metals for a normal stellar cluster is $\sim 0.025$ (Maeder, 1992). Simulations of the dynamical evolution of H I gas around NGC 1533 show that it could last up to $1 \times 10^{9}$ yrs (Ryan-Weber 2003a). This is considered an upper limit since no consumption of gas due to the formation of stars is taken into account. If the SFR is maintained for $1 \times 10^{9}$ 
yrs, metals will pollute the $2.4 \times 10^{9} \mathrm{M}_{\odot}$ of $\mathrm{H} \mathrm{I}$ in the $\mathrm{SE}$ cloud, resulting in a metallicity of $\sim 1-2 \times 10^{-3}$ solar. Alternatively if the SFR was not continuous and corresponded to a single population of stars only, the resulting metallicity would be negligible $\left(\sim 4 \times 10^{-6}\right.$ solar $)$.

How does this compare to the abundances seen in Ly $\alpha$ absorption line systems? $\mathrm{HI}$ in the vicinity of the NGC 1533 isolated H II regions has $N_{\mathrm{HI}}$ $=1-4 \times 10^{20} \mathrm{~cm}^{-2}$, similar to damped Ly $\alpha$ absorption (DLA, $N_{\mathrm{HI}} \geq 2 \times 10^{20}$ $\mathrm{cm}^{-2}$ ). The metallicity of DLA gas at low redshift varies from 0.01 solar (e.g I Zw 18, Aloisi et al. 2003) to solar. Depending on the initial metallicity, the isolated H II regions would enrich the NGC 1533 system by 20 percent at the most. At higher redshifts however, this increase in metallicity could be more significant. Prochaska et al. (2003) find a DLA 'metallicity floor' at $\sim 1.4 \times 10^{-3}$ solar, over a redshift range from 0.5 to 5 . Intergalactic star formation may have contributed to this. In addition, since collisions and tidal disruptions of galaxies were more common at higher redshifts, the amount of high $N_{\mathrm{HI}}$-gas outside galaxies was greater and therefore the intergalactic star formation rate could have been higher in the past.

Acknowledgments. Thanks to the SINGG team for the images. This research has made use of the NASA/IPAC Extragalactic Database (NED). Digitized Sky Survey (DSS) material (UKST/ROE/AAO/STScI) is acknowledged.

\section{References}

Aloisi, A., Savaglio, S., Heckman, T.M., Hoopes, C.G., Leitherer, C., \& Sembach, K.R. 2003, ApJ, 595, 760

Collins, J.A., Shull, J.M., \& Giroux, M.L. 2003, ApJ, 585, 336

Ferguson, A.M.N., Gallagher, J.S., \& Wyse, R.F.G. 1998a, AJ, 116, 673

Ferguson, A.M.N., Wyse, R.F.G., Gallagher, J.S., \& Hunter, D.A. 1998b, ApJ, 506, L19

Gerhard, O., Arnaboldi, M., Freeman, K.C., \& Okamura, S. 2002, ApJ, 580, 121

Kennicutt, R.C. 1998, ApJ, 498, 541

Maeder, A. 1992, A\&A, 264, 105

Meyer M., et al. 2003, MNRAS, submitted

Oosterloo, T., et al. 2003, these proceedings

Prochaska, J.X. 2003, ApJ, 582, 49

Ryan-Weber, E., Webster, R., \& Bekki, K. 2003a, in ASSL Vol. 281: The IGM/Galaxy Connection. The Distribution of Baryons at $\mathrm{z}=0$, p. 223

Ryan-Weber, E.V., Webster, R.L., \& Stavely-Smith, L. 2003b, MNRAS, 343, 1195

Ryan-Weber, E.V., et al. 2003c, AJ, submitted

Sakai, S., Kennicutt, R.C., van der Hulst, J.M., \& Moss, C. 2002, ApJ, 578, 842

Tripp, T.M., et al. 2002, ApJ, 575, 697

Vacca, W.D., Garmany, C.D., \& Shull, J.M. 1996, ApJ, 460, 914

Verdes-Montenegro, L., Yun, M.S., Williams, B.A., Huchtmeier, W.K., Del Olmo, A., \& Perea, J. 2001, A\&A, 377, 812 